\title{
Article \\ Tunable Electronic Properties of Few-Layer Tellurene under In-Plane and Out-of-Plane Uniaxial Strain
}

\author{
Genwang Wang ${ }^{1,2}$, Ye Ding ${ }^{1,2}$, Yanchao Guan ${ }^{1,2}$, Yang Wang ${ }^{1,2, *}$ and Lijun Yang ${ }^{1,2, *}$ \\ 1 Key Laboratory of Microsystems and Microstructures Manufacturing, Ministry of Education, Harbin Institute \\ of Technology, Harbin 150001, China; nisker@163.com (G.W.); dy1992hit@hit.edu.cn (Y.D.); \\ guanyanchaoo@163.com (Y.G.) \\ 2 School of Mechatronics Engineering, Harbin Institute of Technology, Harbin 150001, China \\ * Correspondence: wyyh@hit.edu.cn (Y.W.); ylitj@hit.edu.cn (L.Y.)
}

Citation: Wang, G.; Ding, Y.; Guan, Y.; Wang, Y.; Yang, L. Tunable Electronic Properties of Few-Layer Tellurene under In-Plane and Out-of-Plane Uniaxial Strain. Nanomaterials 2022, 12, 875. https://doi.org/10.3390/ nano12050875

Academic Editor: Filippo

Giannazzo

Received: 15 February 2022

Accepted: 4 March 2022

Published: 6 March 2022

Publisher's Note: MDPI stays neutral with regard to jurisdictional claims in published maps and institutional affiliations.

Copyright: (C) 2022 by the authors. Licensee MDPI, Basel, Switzerland. This article is an open access article distributed under the terms and conditions of the Creative Commons Attribution (CC BY) license (https:// creativecommons.org/licenses/by/ $4.0 /)$.

\begin{abstract}
Strain engineering is a promising and fascinating approach to tailoring the electrical and optical properties of 2D materials, which is of great importance for fabricating excellent nanodevices. Although previous theoretical works have proved that the monolayer tellurene has desirable mechanical properties with the capability of withstanding large deformation and the tunable band gap and mobility conductance induced by in-plane strain, the effects of in-plane and out-of-plane strains on the properties of few-layer tellurene in different phases should be explored deeply. In this paper, calculations based on first-principles density functional theory were performed to predict the variation in crystal structures and electronic properties of few-layer tellurene, including the $\alpha$ and $\beta$ phases. The analyses of mechanical properties show that few-layer $\alpha$-Te can be more easily deformed in the armchair direction than $\beta$-Te owing to its lower Young's modulus and Poisson's ratio. The $\alpha$-Te can be converted to $\beta$-Te by in-plane compressive strain. The variations in band structures indicate that the uniaxial strain can tune the band structures and even induce the semiconductor-to-metal transition in both few-layer $\alpha$-Te and $\beta$-Te. Moreover, the compressive strain in the zigzag direction is the most feasible scheme due to the lower transition strain. In addition, few-layer $\beta$-Te is more easily converted to metal especially for the thicker flakes considering its smaller band gap. Hence, the strain-induced tunable electronic properties and semiconductor-to-metal transition of tellurene provide a theoretical foundation for fabricating metal-semiconductor junctions and corresponding nano-devices.
\end{abstract}

Keywords: tellurene; strain engineering; density functional theory; semiconductor-to-metal transition

\section{Introduction}

Since a variety of two-dimensional (2D) materials have been synthesized by mechanical exfoliation, chemical vapor deposition (CVD), physical vapor deposition (PVD), molecular beam epitaxy (MBE), pulsed laser deposition (PLD), and so on [1,2], the mechanical, electrical, optical, and quantum properties make them attractive for applications in transistors, photodetectors, cells, sensors, and memristors [3-6]. However, despite the promising superiority of $2 \mathrm{D}$ materials, their intrinsic properties make them unsuitable for fabricating nano-devices with excellent properties and stability. Hence, methods of tailoring intrinsic properties, such as doping [7,8], electric field tuning $[9,10]$, surface modification [11], and building van der Waals heterostructure [12,13], have become critical ways to improve the performance further. In particular, strain engineering emerges as a perfect candidate for controlling the performance of nano-devices, since the 2D materials are capable of withstanding large in-plane and out-of-plane strains before the rupture $[14,15]$. Moreover, strain can modulate the mechanical, electrical, and optical properties of 2D materials, like phase stability [16], band structure [17], mobility [18], Fermi level [19], and thermal conductivity [20], so that the strain-induced effects provide a fertile library for controlling the properties of advanced nano-devices. For example, the local strain 
field, which is generated from engineering the substrate's surface morphology, leads to a two-orders-of-magnitude increase in the carrier mobility of $\mathrm{MoS}_{2}$ transistors, compared to the conventional devices [21]. Tensile strains in monolayer (ML) $\mathrm{MoS}_{2}$ dramatically enhanced the sensitivity and response performance of a sensor for gas detection by tuning the Schottky barrier height [22]. In addition, the deformation of 2D materials also introduces new phenomena, like piezotronics and piezophototronics, which have potential applications in energy harvesting, flexible, and self-powered nano-devices [23]. Therefore, the exploration of strain engineering in $2 \mathrm{D}$ materials is promising and urgent for future multifunctional nano-devices.

Recently, the existence of 2D tellurium (tellurene) has been predicted by first-principles density functional theory (DFT) calculations [24-26]. These results show that there are more than five stable or meta-stable phases for tellurene, which exhibit semiconductor and metallic properties. Of these, at least two phases, $\alpha$-Te and $\beta$-Te (since the names of different phases in different papers are still not unified, the definition of tellurene phases in this paper follows Refs. [25,26]) were synthesized by solution-grown methods [27], liquid-phase exfoliation [28], MBE [29], PLD [30], and PVD [31]. The tellurene has demonstrated high carrier mobility, high on/off ratio, considerable responsivity, and excellent air stability for applications in FET, photodetectors, and sensors [27,32,33]. Tellurene is also a promising candidate for strain engineering. For instance, according to DFT calculations, ML $\beta$-Te has a low Young's modulus ( $27 \mathrm{GPa})$ and large tensile strain limit $(>30 \%)$, suggesting that it is a kind of ductile material with the capability of withstanding large stretch deformation [34,35]. Moreover, the band structure and mobility of ML $\beta$-Te can also be tuned by the strain $[35,36]$. Experiments also confirmed the strain-induced effects on the electrical properties of tellurene and proved the capacity for developing flexible devices [37-39]. Recent calculation works mainly focused on the in-plane deformation of ML $\beta$-Te. However, the ML tellurene is still hardly acquired by mechanical exfoliation and solution-grown method for the purposes of experiments, compared to graphene, phosphorene, and hexagonal boron nitride. Therefore, the effects of strain on few-layer and multi-layer tellurene, including the crystal structures and mechanical and electrical properties, should be investigated for the design of nano-devices. Furthermore, strain-effects on different phases also offer diverse ways to select suitable materials and methods of controlling properties. Besides the in-plane strain, the out-of-plane strain induced by normal strain and bending deformation also provides another unique means to design and control the electron transport and electron-phonon coupling process [40,41]. Thus, a deeper insight into the profound impact of in-plane and out-of-plane strain on few-layer Te is indispensable and promising for next-generation nano-devices.

In this work, the strain engineering in few-layer $\alpha$-Te and $\beta$-Te was investigated theoretically. First of all, the crystal structures of few-layer $\alpha$-Te and $\beta$-Te were acquired from the bulk tellurium. Calculation results show the main difference between the two phases is the location of the mid-Te atoms. Then, the mechanical properties of unstrained tellurene were calculated, including Young's modulus (YM) and Poisson's ratio (PR). The elasticities of the few-layer $\alpha$-Te and $\beta$-Te were discussed. In addition, the phase transition between the few-layer $\alpha$-Te and $\beta$-Te under the uniaxial strain was investigated by calculating the location of mid-Te atoms. The intrinsic band structures of the few-layer $\alpha$-Te were also calculated to show the variations as the thickness increases. Sequentially, the strain engineering of the electronic properties of tellurene was performed by gradually applying uniaxial strain in the armchair (AC), zigzag (ZZ), and normal (NM) directions. The semiconductor-to-metal transition (SMT) in tellurene was shown during some deformation processes. Therefore, the variation in band structures and density of states under the strain were calculated to reveal the mechanism of the process of SMT. The calculation results in this paper provide a foundation to design promising nano-devices based on tellurene. 


\section{Methods}

All calculations were performed by QUANTUM ESPRESSO based on first-principles density functional theory (DFT) $[42,43]$. The generalized gradient approximation of the Perdew-Burke-Ernzerhof (PBE) exchange correlation functional and projector augmentedwave (PAW) pseudopotential were adopted [44]. The cut-off energy was set to $500 \mathrm{eV}$ after testing different values. In order to avoid the interaction between flakes and its periodic images, the vacuum space of $20 \AA$ was introduced along the out-of-plane direction. All structures were relaxed until the energy in electronic self-consistent field (SCF) iterations and the ionic Hellmann-Feynman forces were lower than $1 \times 10^{-6} \mathrm{eV} /$ atom and $0.01 \mathrm{eV} / \AA$, respectively. In addition, the van der Waals correction proposed by Grimme [45] was considered. During all the calculation processes, a dense k-mesh of $11 \times 15 \times 1$ sampling was used. For the calculations of electronic properties, the spin-orbit coupling (SOC) effect was also included to obtain accurate band structures. To simulate the uniaxial in-plane and out-of-plane deformations of few-layer Te, the tensile and compressive strain were gradually exerted with a step of $1 \%$ in the AC, ZZ, and NM directions. For in-plane strain, the lattice constants were fixed in the out-of-plane and strained directions and atoms were fully relaxed in all directions. During the deformation in the NM direction, the movements of the top and bottom atoms were fixed in the NM direction and relaxed in other directions, while other atoms were relaxed in all directions. Meanwhile, the lattice constant in the NM direction was fixed and the lattice constants along in-plane directions were relaxed.

In order to reveal the structural stability of few-layer tellurene with different phases, the formation energy $\Delta E$ was calculated. The equation was defined as:

$$
\Delta E=E_{\text {tellurene-energy/atom }}-E_{\text {bulk-energy/atom }}
$$

where $E_{\text {tellurene-energy/atom }}$ and $E_{\text {bulk-energy/atom }}$ are the total energy per atom of tellurene and bulk tellurium, respectively.

To investigate the mechanical properties, the orientation-dependent YM E $(\theta)$ and PR $v(\theta)$ were calculated as [46]

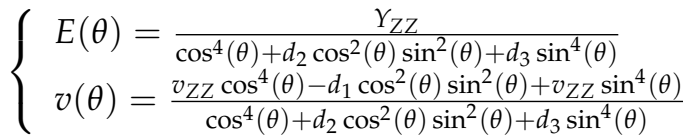

where $d_{1}, d_{2}, d_{3}, Y_{\mathrm{ZZ}}$, and $v_{\mathrm{ZZ}}$ are elastic constants, for $2 \mathrm{D}$ materials, which are defined as

$$
\left\{\begin{array}{l}
v_{\mathrm{ZZ}}=\frac{C_{12}}{C_{22}}, \quad Y_{\mathrm{ZZ}}=\frac{C_{11} C_{22}-C_{12}^{2}}{C_{22}} \\
d_{1}=\frac{C_{11}}{C_{22}}+1-\frac{C_{11} C_{22}-C_{12}^{2}}{C_{22} C_{66}}, \quad d_{2}=-\left(2 \frac{C_{12}}{C_{22}}-\frac{C_{11} C_{22}-C_{12}^{2}}{C_{22} C_{66}}\right), \quad d_{3}=\frac{C_{11}}{C_{22}}
\end{array}\right.
$$

where $C_{11}, C_{12}, C_{22}$, and $C_{66}$ are elastic stiffness constants, which can be obtained based on the strain energy and Hooke's law [47], shown as:

$$
\begin{gathered}
{\left[\begin{array}{l}
\sigma_{11} \\
\sigma_{22} \\
\sigma_{12}
\end{array}\right]=\left[\begin{array}{lll}
C_{11} & C_{12} & 0 \\
C_{12} & C_{22} & 0 \\
0 & 0 & C_{66}
\end{array}\right]\left[\begin{array}{c}
\varepsilon_{11} \\
\varepsilon_{22} \\
2 \varepsilon_{12}
\end{array}\right]} \\
C_{i j}=\frac{1}{A\left(\varepsilon_{i j}\right)}\left(\frac{\partial E^{2}}{\partial \varepsilon_{i} \partial \varepsilon_{j}}\right)
\end{gathered}
$$

where $E$ is the strain energy and $A\left(\varepsilon_{\mathrm{ij}}\right)$ is the area under the strain. $\varepsilon$ is the applied strain, which is defined as $\varepsilon=\left(a-a_{0}\right) / a_{0}$, where $a$ and $a_{0}$ are the lattice constants of strained and un-strained structures, respectively. The range of applied strain for calculating elastic stiffness constants is from $-2 \%$ to $2 \%$ with a step of $0.5 \%$. 


\section{Results and Discussion}

\subsection{Crystal Structures}

The bulk tellurium, which belongs to the space group $\mathrm{P}_{3} 21$, has helical chains of Te atoms that are stacked together by non-covalent bonding at the center and corners of the hexagonal cell. Figure $1 \mathrm{a}, \mathrm{b}$ present the top and side views of the atomic configurations. The optimized lattice constants of the bulk Te are $a=b=4.34 \AA, c=6.03 \AA$, which are consistent with previous works [24]. Then, for few-layer $\alpha$-Te, for instance, the bilayer (BL) shown

in Figure 1c,d, can be obtained by cutting the bulk Te along the parallel $\{10 \overline{10}\}$ planes (the blue solid lines in Figure 1a,b). In the $\alpha$-Te, the Te atoms still maintain the chain structures along the $x$-direction. However, compared to $\alpha$-Te, the $\beta$ phase can be regarded as a kind of symmetric structure by moving the mid-Te atoms (the red atoms $\mathrm{Te}_{1}$ and $\mathrm{Te}_{4}$ ) to the symmetric position (red dotted circles), presented in Figure 1e,f. The mid-atoms are not only bonded to the atoms in same chains but also the adjacent chains. Therefore, the main difference between the $\alpha$-Te and $\beta$-Te is the locations of the mid-Te atoms. Hence, in this paper, the fractional distance, which was defined as $r=b_{\mathrm{m}} / b$, was used to indicate the $\alpha$ and $\beta$ phases. $b_{\mathrm{m}}$ is the average distance along the $y$ axis between the mid-Te atom and other two atoms in the same Te chain, as shown in Figure 1c. For the $\beta$-Te, the mid-Te atoms are located at the symmetric position, so that the fractional distance $r$ equals 0.5 . However, the $\alpha$-Te has an $r$ value less than 0.5 since the mid-Te atoms are far from the adjacent chains. The lattice parameters and formation energy $\Delta E$ of $\alpha$-Te and $\beta$-Te with BL, three-layer (TL), and four-layer (FL) are presented in Table 1. According to the crystal structures of ML Te, the $\alpha$ phase is not stable and transforms into $\beta$. This phenomenon has been concluded by other theoretical works $[25,26]$. For the few-layer tellurene, crystal structures of both $\alpha$ and $\beta$ phase were acquired. Despite the positive formation energy, which means the bulk tellurium is more stable than layered, the relative lower values (for instance, the formation energy of $\mathrm{MoS}_{2}$, silicene, and germanene are $0.28 \mathrm{eV} / \mathrm{atom}$, $0.76 \mathrm{eV} /$ atom, and $0.99 \mathrm{eV} /$ atom, respectively $[48,49]$ ) also give support to the existence of few-layer $\alpha$-Te and $\beta$-Te, especially for the thicker flakes. The previous experiments and phonon spectra in calculations have also proved the existence of both phases [25-31]. In addition, the small differences in formation energy between the $\alpha$ and $\beta$ phases mean the possibility of transition between them. This phase transition has also been predicted by calculation works $[50,51]$. Hence, due to the differences in the crystal structures, the mechanical and electronic properties of few-layer $\alpha$-Te and $\beta$-Te and the strain engineering in them should be investigated separately.

\subsection{Mechanical and Electronic Properties}

Before exploring the strain effect on the properties of few-layer $\alpha$-Te and $\beta$-Te, the mechanical properties were firstly glimpsed, including elastic stiffness constants, YM, and PR. Table S1 shows the calculated results. For ML and few-layer Te, the elastic stiffness constants satisfy the equilibrium conditions $\left(C_{11} C_{22}-C_{12}{ }^{2}>0\right.$, and $\left.C_{11}, C_{66}>0\right)$, which proves the mechanical stability of all crystal structures. Figure S1 presents the orientationdependent YM and PR of ML $\beta$-Te. The maximal YM is $24.8 \mathrm{~N} / \mathrm{m}$ along the $\mathrm{ZZ}$ direction and the minimal is $11.1 \mathrm{~N} / \mathrm{mm}$ for the AC direction. The corresponding PR values are 0.59 and 0.26 along the $\mathrm{ZZ}$ and $\mathrm{AC}$ directions, respectively. These results, which are similar to the previous works $[34,35]$, indicate that the ML $\beta$-Te has a stronger anisotropy of mechanical properties and is more easily deformed in the AC direction than in the $\mathrm{ZZ}$ direction. However, for few-layer tellurene, $\alpha$ and $\beta$ phases exhibit significant differences in YM and PR. Figure 2 shows the orientation-dependent YM and PR of TL tellurene (the BL and FL tellurene are shown in Figure S2). In order to investigate the effect of uniaxial strain on electronic properties, YM and PR in the direction of $\mathrm{AC}\left(\theta=0^{\circ}\right)$ and $\mathrm{ZZ}\left(\theta=90^{\circ}\right)$ are mainly focused upon. Except for the BL flakes, $\alpha$-Te has smaller YM and PR along the ZZ direction than $\mathrm{AC}$, which can be attributed to the bonding atom chains in the AC direction but weak van-der-Waals-type bonds between chains (ZZ direction), as shown in Figure 1c,d. The performance of few-layer $\beta$-Te is analogous to ML $\beta$-Te. Compared to $\alpha$-Te, the values 
of $\mathrm{YM}$ and PR are slightly decreased along the AC direction. However, few-layer $\beta$-Te has larger YM and PR along the ZZ direction. Referring to the crystal structures shown in Figure 1e,f, mid-Te atoms are not only bonded to other atoms in same chains but also the atoms in adjacent chains in the $\beta$ phase, so that deformation along the ZZ direction requires a larger force to overcome the bond energy. Conclusively, few-layer $\alpha$-Te and $\beta$-Te have inverse anisotropic elastic properties, which are dominated by the location of mid-Te atoms. However, $\alpha$-Te is more sensitive to the strain than $\beta$-Te in the ZZ direction, which is beneficial for tuning electrical properties by strain.

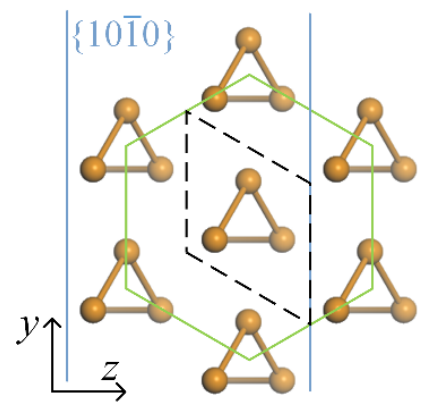

(a)

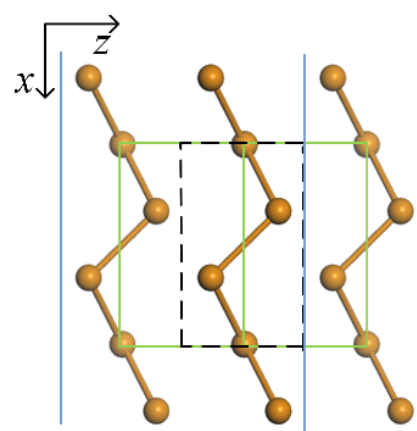

(b)

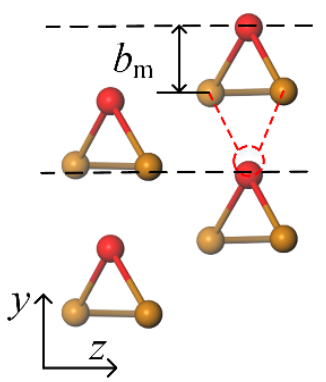

(c)

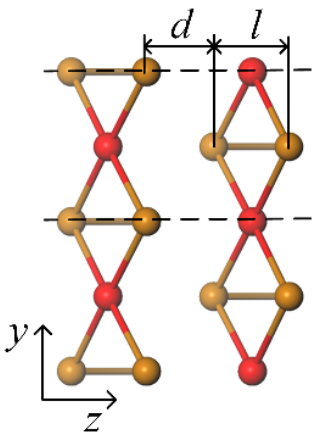

(e)

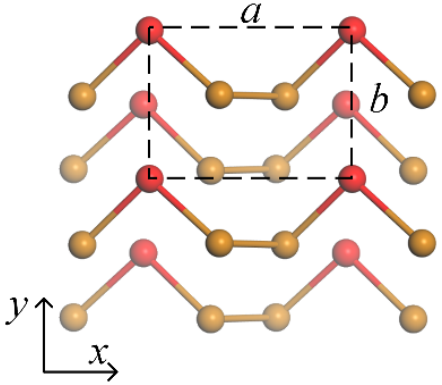

(d)

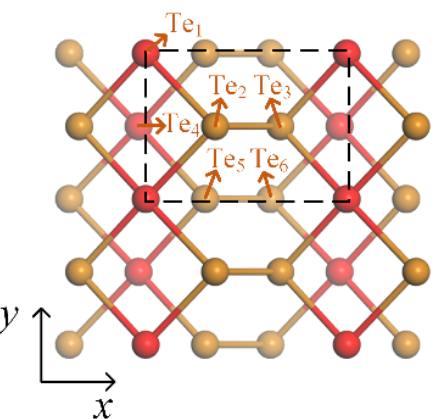

(f)

Figure 1. Atomic configurations of the bulk Te, BL $\alpha$-Te, and BL $\beta$-Te. Top (a) and side (b) views of bulk Te. The green and blue solid lines in $(\mathbf{a}, \mathbf{b})$ show the hexagonal element cell and $\{10 \overline{10}\}$ planes of bulk Te, respectively. Side (c) and top (d) views of BL $\alpha-$ Te, side (e) and top (f) views of BL $\beta$-Te. $a$ and $b$ are the lattice constants along the $x$ - and $y$-directions. $l$ and $d$ represent the average of layer thickness and interlayer distance, respectively. The red dotted lines and circles show the symmetric position of the red Te atom and bonds in the $\beta$ phase. The black dotted lines in $(\mathbf{a}-\mathbf{f})$ show the unit cell in the DFT calculations.

Table 1. Lattice parameters and formation energy $\Delta E$ of $\alpha$-Te and $\beta$-Te.

\begin{tabular}{cccccccc}
\hline Phase & $\begin{array}{c}\text { Layer } \\
\text { Number }\end{array}$ & $\boldsymbol{a}(\AA)$ & $\boldsymbol{b}(\AA)$ & $\boldsymbol{l}(\AA)$ & $\boldsymbol{d}(\AA)$ & $\boldsymbol{\Delta})$ & $\begin{array}{c}\Delta E \\
\text { (eV/atom) }\end{array}$ \\
\hline \multirow{4}{*}{$\alpha-\mathrm{Te}$} & ML & 5.49 & 4.17 & 2.16 & $/$ & 0.50 & 0.228 \\
& BL & 5.80 & 4.24 & 2.10 & 1.79 & 0.43 & 0.134 \\
& TL & 5.89 & 4.28 & 2.07 & 1.77 & 0.42 & 0.095 \\
& FL & 5.92 & 4.30 & 2.08 & 1.75 & 0.42 & 0.073 \\
\hline \multirow{3}{*}{$\beta-\mathrm{Te}$} & ML & 5.49 & 4.17 & 2.16 & 1 & 0.50 & 0.228 \\
& BL & 5.78 & 4.18 & 2.1 & 1.88 & 0.50 & 0.137 \\
& TL & 5.89 & 4.18 & 2.05 & 1.87 & 0.50 & 0.101 \\
& FL & 5.94 & 4.19 & 2.04 & 1.83 & 0.50 & 0.081 \\
\hline
\end{tabular}




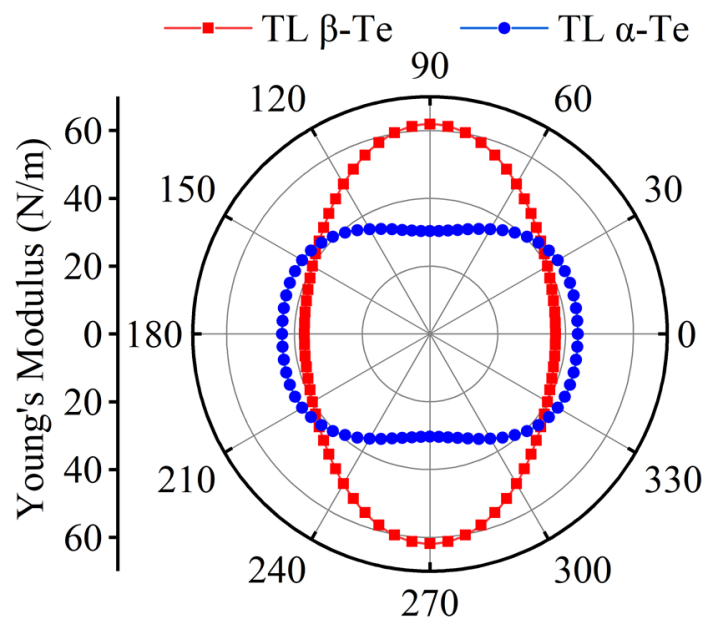

(a)

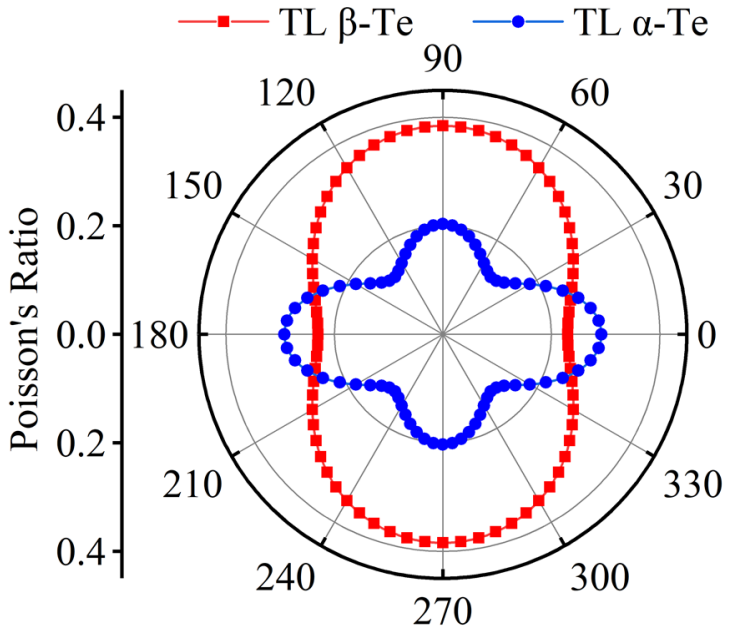

(b)

Figure 2. Mechanical properties of TL $\alpha$-Te and $\beta$-Te. (a) Orientation-dependent YM, (b) orientationdependent PR.

As mentioned in other calculation works, the transition between the $\alpha$ and $\beta$ phases is feasible [26,50]. To give an insight into the phase transition, the variations in atom structures under uniaxial strain in the range $-14 \% \leq \varepsilon \leq 14 \%$ were calculated. Despite compressive and tensile strains applied on $\beta$-Te in $\mathrm{AC}, \mathrm{ZZ}$, and NM directions, the fractional distance $r$ is still maintained at 0.5 , which means no phase transition from $\beta$-Te to $\alpha$-Te occurs during uniaxial deformation. However, as Figure 3 shows, few-layer $\alpha$-Te can be transformed to the $\beta$ phase under compressive strain in the AC or ZZ directions and will maintain the $\beta$ phase even if the compressive strain increases. The critical strains of phase transition for BL, TL, and FL $\alpha$-Te are about $-7 \%,-11 \%$, and $-13 \%$, respectively, in the $\mathrm{AC}$ direction, and $-5 \%,-9 \%$, and $-9 \%$ in the $\mathrm{ZZ}$ direction. The lower critical strain in the $\mathrm{ZZ}$ direction is consistent with the lower $\mathrm{YM}$ in the $\mathrm{ZZ}$ direction and the higher PR in the AC direction, which confirms the capacity of uniaxial strain to change atom structures. In addition, thicker flakes require higher strain for the phase transition due to higher deformation resistibility. When the tensile strain applies along the AC and ZZ directions, the decreased fractional distances indicate the persistence of the $\alpha$ phase, which means no phase transition occurs. For the normal strain shown in Figure $3 c$, despite BL $\alpha$-Te approximately transforming to $\beta$-Te when the tensile strain attains $14 \%$, fractional distances of TL and FL only increase slightly under tensile strain and no phase transition is shown at least in the range $0 \leq \varepsilon \leq 14 \%$. Furthermore, the impact of tensile strain on fractional distances becomes smaller as the thickness increases. Hence, according to the results, the compressive strain in the $\mathrm{ZZ}$ direction is more suitable to induce the phase transition from $\alpha$ to $\beta$ due to lower required strain $(-5--9 \%)$. Previous calculations have also shown that the strain limits of ML tellurene attain $-24 \%$ and $-22 \%$ in the AC and $\mathrm{ZZ}$ directions [35]. Although no calculations and experiments have proved the strain limits of few-layer tellurene so far, the compressive strains in the ZZ direction still have the potential to induce phase transition before the fracture of materials. The strain-induced phase transition and the corresponding application should be further confirmed in experiments. In addition, attention should be paid to the phase transition during the tuning of electronic properties by the strain. 




(a)

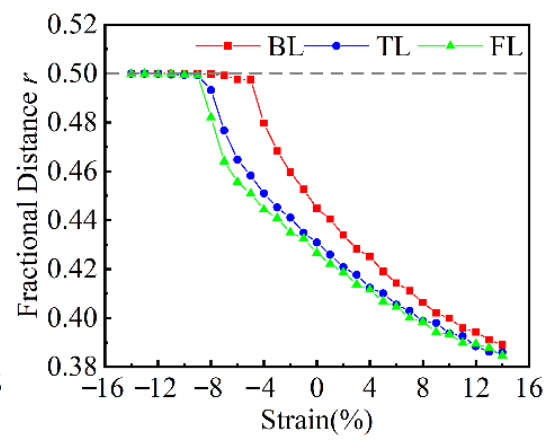

(b)



(c)

Figure 3. Fractional distances of few-layer $\alpha$-Te versus uniaxial strain in different directions. (a) AC direction; (b) ZZ direction; (c) NM direction.

Since the $\alpha$-Te and $\beta$-Te present the different performances of mechanical properties, the electronic properties of these two phases also need to be compared. The Brillouin zone used in the calculations and the results of these are shown in Figure S3; the ML $\beta$-Te is a semiconductor 2D material with direct bandgaps of $1.04 \mathrm{eV}$ and $1.46 \mathrm{eV}$ calculated by PBE + SOC and HSE (hybrid functional) +SOC schemes, respectively, which are consistent with previous works $[24,35]$. The valence band maximum (VBM) and conduct band minimum (CBM) of ML $\beta$-Te are both located at the $\Gamma$ point and determined by the $p_{y}$ and $p_{x}$ orbitals of Te, respectively, and the total density of states (TDOS) and projected density of states (PDOS) are shown in Figure S3d. These suitable direct band gaps reveal that the ML $\beta-T e$ is a promising 2D material for application in electrical and optical nanodevices. The band structures of few-layer tellurene are shown in Figure 4 and Figure S4. The band gaps of both $\alpha$ and $\beta$ phases decay as the thickness increases; these are $0.82 \mathrm{eV}$ (BL), $0.62 \mathrm{eV}$ (TL), and $0.46 \mathrm{eV}$ (FL) for $\alpha$-Te and $0.33 \mathrm{eV}$ (BL), $0.07 \mathrm{eV}$ (TL), and $0.03 \mathrm{eV}$ (FL) for $\beta$-Te. Apparently, the $\beta$ phase has a smaller band gap than $\alpha$ for the same thickness. Moreover, the $\alpha$-Te with different layers exhibits variation in VBM and CBM. While ML Te has a direct band gap at the point of $\Gamma, \mathrm{BL} \alpha$-Te shows an indirect band gap as the CBM moves to the point between $\Gamma$ and $X$, as shown in Figure S4a. However, the VBM and CBM shift to around the point of $S(0.50,0.32,0)$ and the TL and FL $\alpha$-Te turn into direct semiconductors again. The few-layer $\beta$-Te still has a direct band gap at the point of $\Gamma$ regardless of the thickness. Hence, comparing to the $\alpha$-Te, the few-layer $\beta$-Te is more easily converted to metal due to the small band gap.

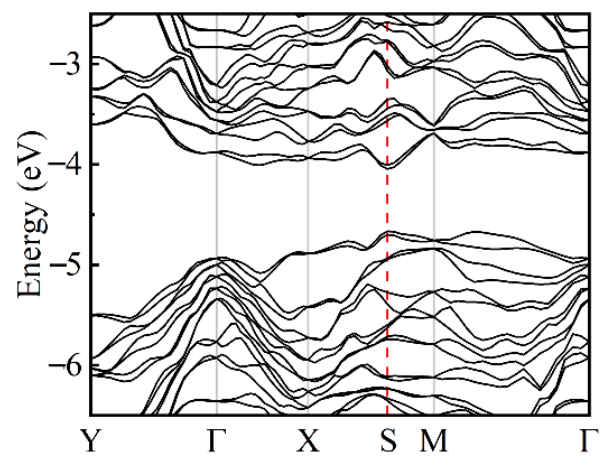

(a)

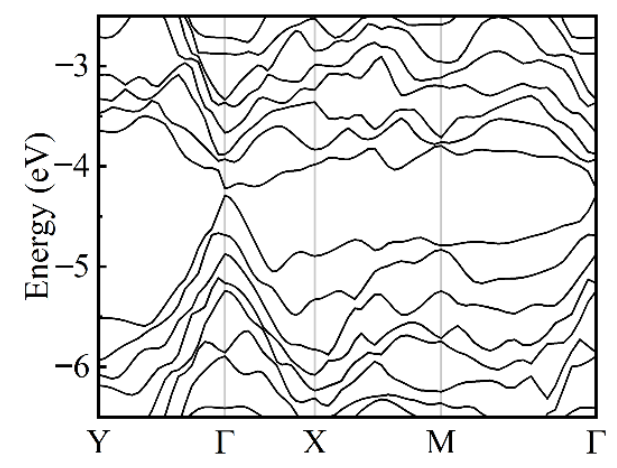

(b)

Figure 4. Band structures of TL tellurene. (a) $\alpha$-Te; (b) $\beta$-Te.

\subsection{Effects of Uniaxial Strain on Electronic Properties}

As the $\alpha$-Te and $\beta$-Te show distinct mechanical and electronic properties, performances of these two phases under uniaxial strain should be discussed to explore the control of 
electronic properties. The strain effects on ML $\beta$-Te were firstly investigated. Figure $5 \mathrm{a}$ shows the variation in band gap when compressive and tensile strains are exerted on the $\mathrm{ML} \beta$-Te along the $\mathrm{AC}$ and $\mathrm{ZZ}$ directions. Under the compressive strains along the $\mathrm{AC}$ direction and the tensile strain along the ZZ direction, the band gap of ML $\beta$-Te slightly varies at first and then decays quickly after a critical strain $(-11 \%$ and $7 \%$, respectively). However, the decrease in band gap is still small as the strains increase to $-14 \%$ and $14 \%$, which means limited effects of these kinds of strain on the band structure. However, when the compressive strain along the $\mathrm{ZZ}$ direction applies, the band gap decreases more quickly than strains in the other direction and induces the SMT with a critical strain of $-11 \%$. In order to investigate the mechanism of the SMT induced by uniaxial strain, the band structures and PDOS were calculated. Figure $5 \mathrm{~b}$ presents the variation in the energy of the VBM and CBM (with respect to vacuum level) during the compression process. The compressive strain not only forces the CBM to drop down to a lower energy, but also pushes the VBM up to a higher energy, which results in the SMT. Moreover, the strain has a greater impact on the energy of VBM rather than CBM. Figure $5 \mathrm{c}$,d show the band structures and the PDOSs of ML $\beta$-Te when the strain is $-1 \%$ and $-6 \%$. Compared to the intrinsic band structure shown in Figure S3b, the CBM shifts from $\Gamma$ to $X$ and the direct band gap suddenly becomes indirect even when small compressive strain $(-1 \%)$ is applied. As the compressive strain increases, the CBM and VBM maintain the location but the gap between them becomes smaller until the transition to metal (see Figure S5). To have a deep insight into the shift of CBM, the PDOSs of ML $\beta$-Te under the compressive strain are illustrated in the right diagrams of Figure $5 c, d$ and Figure S5. Compared to the unstrained ML $\beta$-Te, the VBM is still determined by the $p_{y}$ orbitals of Te. However, the CBM is mainly contributed by $p_{z}$ instead of $p_{x}$ orbitals. Figure $S 6$ gives the PDOSs of every Te atom in the ML $\beta$-Te under compressive strain of $-6 \%$. It is apparent that the $\mathrm{p}_{\mathrm{z}}$ orbitals are contributed from the two middle Te atoms in the unit cell $\left(\mathrm{Te}_{2}\right.$ and $\mathrm{Te}_{2}$ in Figure $1 \mathrm{f}$ and Figure S3a). Therefore, the compressive strain along the $\mathrm{ZZ}$ direction drives the two atoms to move closer to each other, resulting in the overlap of $p_{z}$ orbitals and the shift in CBM. Hence, the compressive strain along the $\mathrm{ZZ}$ direction tunes the energy and location of VBM and CBM and enables the semiconducting ML $\beta$-Te to convert to metal. Besides the compressive strain along the $\mathrm{ZZ}$ direction, the tensile strain along the AC direction also results in a decrease in the band gap. However, no SMT in ML $\beta$-Te is observed until the strain increases to $14 \%$. Figure S7 shows the details of band structures in this case. The ML $\beta$-Te changes to a semiconductor with an indirect bad gap (the CBM shifts to the point between $X$ and $M$ ) when the tensile strain attains $6 \%$ and become a metal when the strain increases to $17 \%$. Although the previous calculation works showed that the tensile strain limit of ML $\beta$-Te in the AC direction is larger than $36 \%[34,35]$, such high strain may cause the plastic deformation of materials and is hardly performed in experiments. Hence, comparing the tensile strain in the AC direction, the compressive strain along the $\mathrm{ZZ}$ direction is more suitable for realizing the SMT. However, it should be noted that the compressive strain of $-11 \%$ is still hard to exert in experiments. Further experimental investigations are indispensable for the application of strain-induced SMT.

The band gaps of few-layer $\alpha$-Te under uniaxial strain along the AC, ZZ, and NM directions are shown in Figure 6. Similar to the ML $\beta$-Te, as the tensile strain applies along the AC direction, the band gap gradually decreases and finally reaches zero. However, the critical strains for the SMT $(13 \%, 11 \%$, and $10 \%$ for BL, TL, and FL, respectively) are smaller than ML $\beta$-Te and tend to be lower as the thickness increases. Although large compressive strain along the AC direction also leads to a narrow band gap, only FL $\alpha$-Te shows SMT in the range $-14 \% \leq \varepsilon \leq 0$. Since the thicker $\alpha$-Te has a lower band gap, it can be concluded that the SMT will also occur when the layer increases. During the deformation along the $\mathrm{ZZ}$ direction, the change in the band gap is minor when applying the tensile strain or small compressive strain, especially for TL and FL $\alpha$-Te. As the compressive strain reaches a certain value, the band gap suddenly decays and disappears quickly. The critical strains for SMT are $\sim-8 \%$ for BL, TL, and FL $\alpha-\mathrm{Te}$, which are lower than the situation of tensile 
strain along the AC direction. Hence, the compressive strain along the $\mathrm{ZZ}$ direction is more suitable for the realization of SMT. Moreover, the required strain for the SMT in few-layer $\alpha$-Te are smaller than ML tellurene. However, the strain along the NM direction cannot result in SMT at least in the range $-14 \% \leq \varepsilon \leq 14 \%$ (the transition strain is $-21 \%$ for FL $\alpha$-Te, as shown in Figure S8), despite the decrease in band gap under compressive strain. In addition, small tensile strain along NM can increase the band gap, which provides a way to tune the electrical and optical properties for the application of nano-devices.

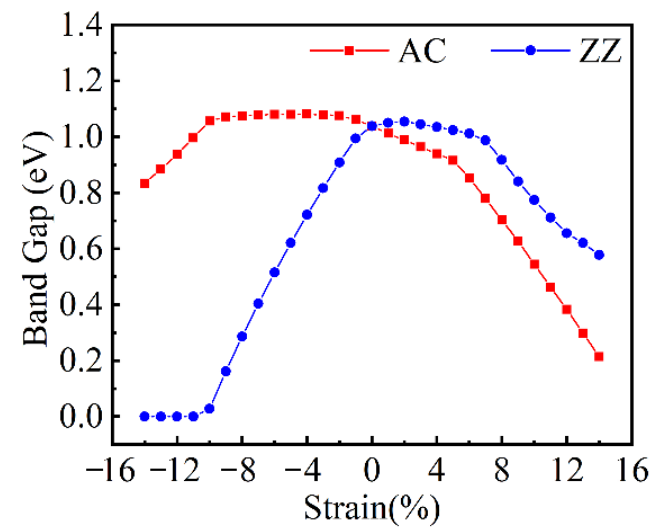

(a)

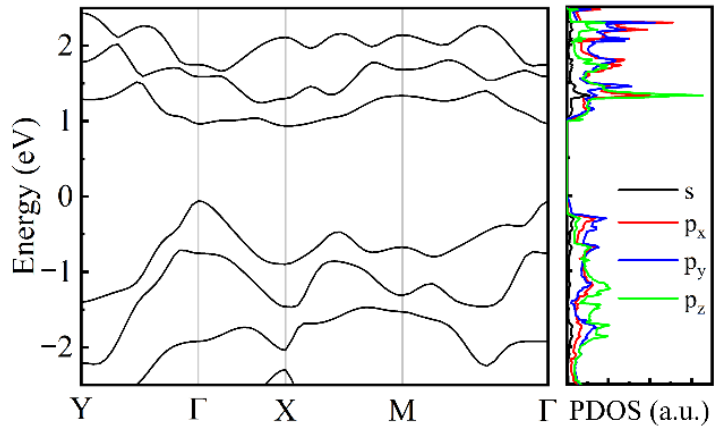

(c)

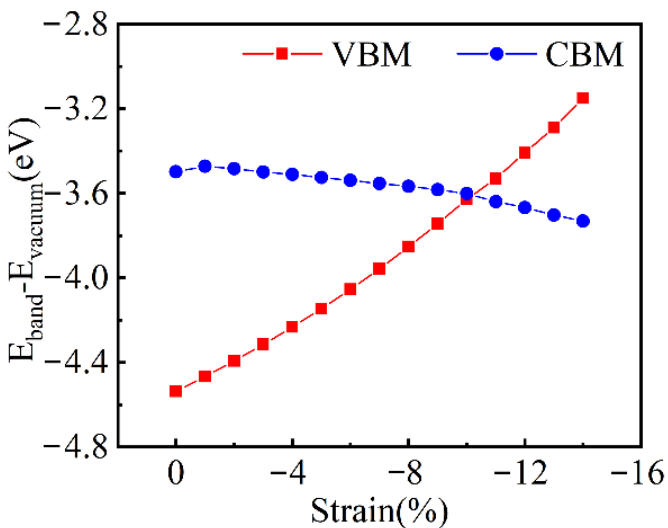

(b)

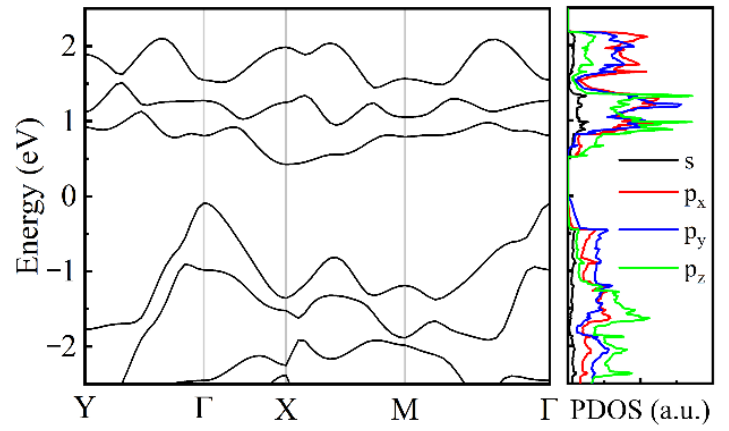

(d)

Figure 5. Electronic properties of ML $\beta$-Te under the in-plane uniaxial strain. (a) The variation in band gap; (b) the changes in the energy of VBM and CBM under the compressive strain along the ZZ direction; band structures (left) and PDOSs (right) under the strains of $-1 \%$ (c) and $-6 \%$ (d) along the $\mathrm{ZZ}$ direction.

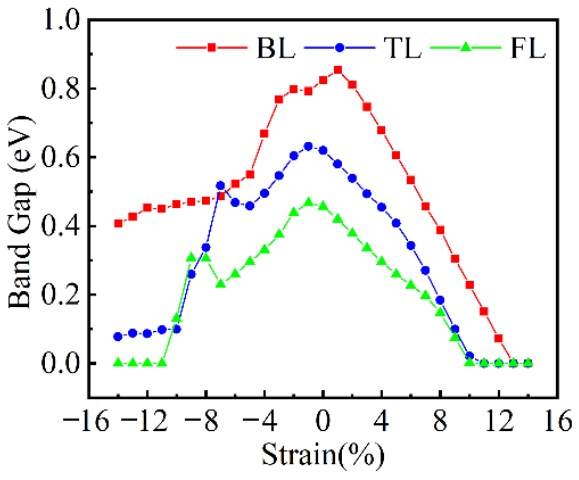

(a)



(b)

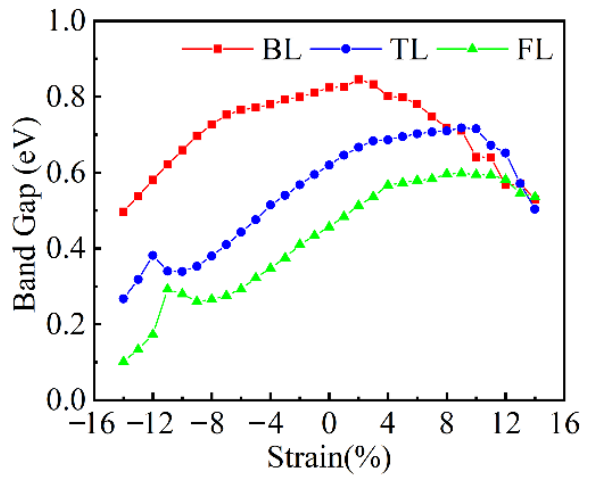

(c)

Figure 6. The variation in band gap of few-layer $\alpha$-Te under uniaxial strain in different directions. (a) AC direction; (b) ZZ direction; (c) NM direction. 
The effects of tensile strain along the AC direction and compressive strain along the ZZ direction on the band structures of few-layer $\alpha$-Te are discussed to gain a deeper insight into the mechanism of strain-induced SMT. During the stretching process in the AC direction, the energy of VBM is sensitive to the deformation and drops down linearly, as shown in Figure $7 \mathrm{a}$. The CBM drops down before the strain increases to around $6 \%$ and then rises up after that. Therefore, this "drop-rise" process of VBM induces a slow followed by a fast decrease in band gap (see Figure 6a). However, for the compression in the ZZ direction, the changes in the energy of CBM and VBM, shown in Figure 7d, are diametrically opposed to the stretching process in the AC direction. The compressive strain slightly puts the CBM down to lower energy before the occurrence of SMT. The VBM goes up quickly after a minor decline. This variation in VBM results in a lower necessary strain for the realization of SMT by contrasting with the stretching scheme. Figure 7 also shows the band structures of TL $\alpha$-Te under some critical strain in these two deformation schemes. The unstrained TL $\alpha$-Te has a direct band gap with CBM and VBM locating at the point of $S(0.50,0.32,0)$, as shown in Figure $4 \mathrm{~b}$. As the tensile strain applies along the AC direction, the band structure still maintains a direct type at first but the energy of the $S$ point gradually decays relatively. When the tensile strain increases to $5 \%$, the VBM shifts to the point of $(0.50,0.18,0)$ and induces an indirect gap, as shown in Figure $7 \mathrm{~b}$. Then, the VBM is moved to near the $\Gamma$ point by further stretching and the energy of it starts to rise up until the occurrence of SMT (see Figure S9a). Therefore, there is a long shift process of VBM with the decrease in energy. In addition, during the whole stretching process, although the strain leads to a large decline in CBM energy, the influence on the location of CBM is small. Figure 7e,f demonstrate the variation in band structure during the compressive strain application. Similar to the tensile strain along the AC direction, the band structures of $\alpha$-Te under the low compressive strain along the ZZ direction are also the direct type. However, the VBM quickly shifts to around the $\Gamma$ point when the strain increases to $-3 \%$ without any transitional location during the process. Before the SMT (see Figure S9b), the locations of VBM and CBM no longer change. However, the energy of VBM dramatically rises, which corresponds to the changes shown in Figure 7d. In addition, unlike the ML $\beta$-Te, during the transition from semiconductor to metal, neither scheme can change the dominant orbitals of Te atoms in the CBM and VBM. The VBM has been determined by the $p_{y}$ and the CBM depends on $p_{y}$ and $p_{z}$ orbitals (see Figure S10). Hence, according to the variation in band structure, although the tensile strain along the AC direction and compressive strain along the ZZ direction both induce the SMT, the intrinsic mechanism is different. The compressive strain along the $\mathrm{ZZ}$ direction is a preferred candidate for making the band gap narrower or resulting in SMT.

With a very small band gap, the FL $\beta$-Te can easily become metal by applying uniaxial strain, referring to Figure S11. To show the effect of strain on few-layer $\beta$-Te, the variations in band gap in BL and TL $\beta$-Te under uniaxial strain were plotted in Figure 8 . For the inplane deformation, except the slight enlargement of band gap induced by small compressive strain along the AC direction, the tensile strain along the AC direction and the deformation in the ZZ direction will result in the SMT. It is apparent that the required strain for SMT is lower for thicker tellurene. To explore the mechanism of SMT in few-layer $\beta$-Te, the BL $\beta$-Te is chosen as an example. Changes in band structures induced by uniaxial strain are shown in Figure 9. The tensile strain along the AC direction slightly puts the VBM down to lower energy and cannot change the location of it. However, the CBM shifts from the location of $\Gamma$ to $X$ with a minor reduction in energy, when the tensile strain increases to $5 \%$, as shown in Figure 9b. The VBM energy decays fast with no changes in the location until the occurrence of SMT, as the strain reaches $10 \%$ (the band structure is shown in Figure S12a). For the deformation in the $\mathrm{ZZ}$ direction, the direct band gap changes to indirect due to little shift of CBM when small tensile and compressive strains apply, as shown in Figure S13. As the tensile strain increases, both the energy of CBM and VBM similarly drop, which indicates that the band gap has a small variation. However, when the strain reaches $7 \%$, as the Figure $9 \mathrm{~d}$ shows, the VBM moves to the point between $X$ and $M$. After that, the energy of VBM starts to increase, which leads to the SMT (the band structure under the 
critical strain of $10 \%$ is shown in Figure S12b). The effect of compressive strain on the band structures is similar to the tensile strain. However, the compressive strain raises the energy of CBM and VBM initially and then puts the CBM down after the location shifts to around the $S$ points (Figure 9f). Comparing the other two schemes, the required compressive strain in the $\mathrm{ZZ}$ direction for the SMT is the lowest. The band structure of the metric BL $\beta$-Te induced by compressive strain of $-7 \%$ is shown in Figure S12c. In addition, the band structure is extremely similar to the BL $\alpha$-Te under the strain of $-8 \%$. This can be attributed to the phase transition from $\alpha$ to $\beta$. Therefore, the phase transition also has effects on the electric properties during the uniaxial deformation. The variations in PDOSs are shown in Figure S15. The CBM and VBM of intrinsic BL $\beta$-Te depend on the py orbitals of Te atoms. In the three deformation methods, only the tensile strain along the $\mathrm{ZZ}$ direction has a significant influence on the CBM and makes the primary PDOS change to $\mathrm{p}_{\mathrm{x}}$, which can be attributed to the strain-induced overlap of $\mathrm{p}_{\mathrm{x}}$ orbitals of the four Te atoms $\left(\mathrm{Te}_{1}, \mathrm{Te}_{2}\right.$, $\mathrm{Te}_{4}$, and $\mathrm{Te}_{6}$ shown in Figure 1f); see Figure S16. Under the strain in the NM direction, the few-layer $\beta$-Te exhibits a similar performance to $\alpha$-Te. The tensile strain is capable of enlarging the band gap and the compressive strain can hardly induce the SMT, despite slightly decreasing the gap. Hence, due to the lower required strain, the compressive strain in the $\mathrm{ZZ}$ direction is also the most applicable way to induce the SMT.

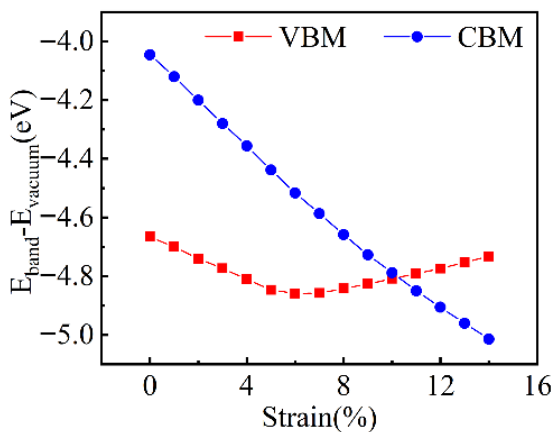

(a)

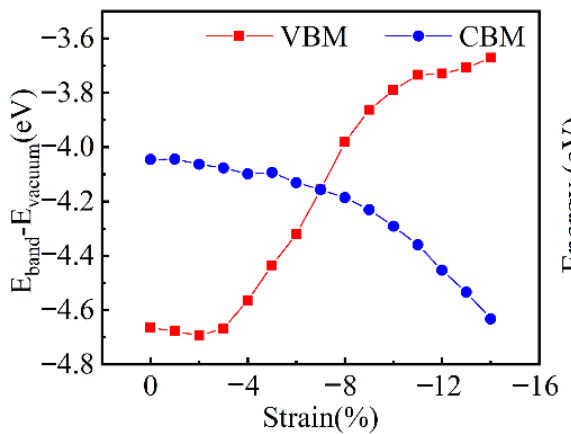

(d)

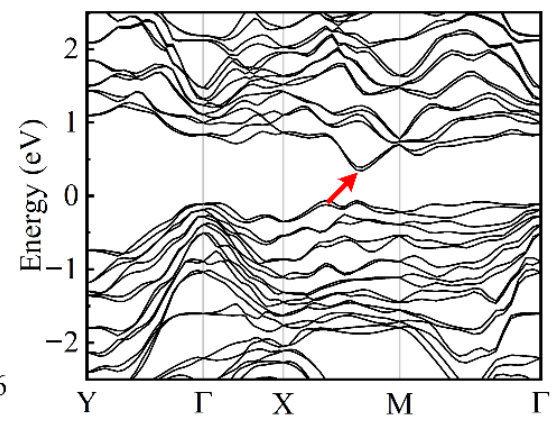

(b)

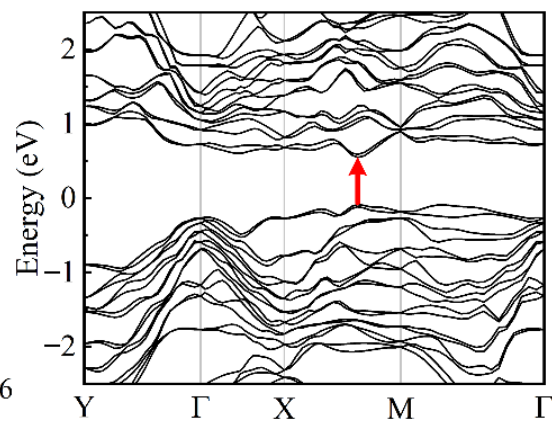

(e)

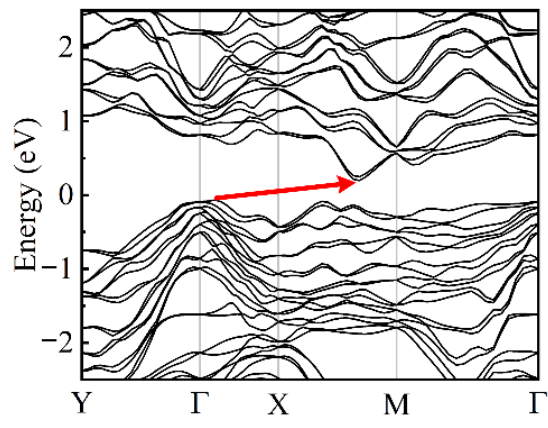

(c)

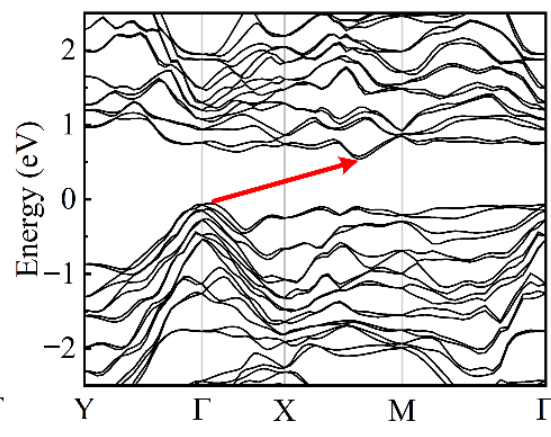

(f)

Figure 7. The variation in band structures of TL $\alpha$-Te under uniaxial strain. The changes in the energy of VBM and CBM with tensile strains along the AC direction (a) and compressive strains along the ZZ direction (d); band structures of TL $\alpha$-Te under tensile strains of $5 \%$ (b) and $7 \%$ (c) along the AC direction and compressive strains of $-1 \%$ (e) and $-3 \%$ (f) along the $\mathrm{ZZ}$ direction. 


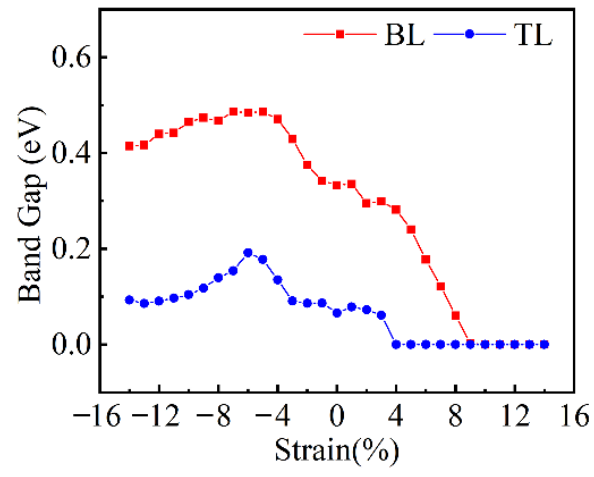

(a)

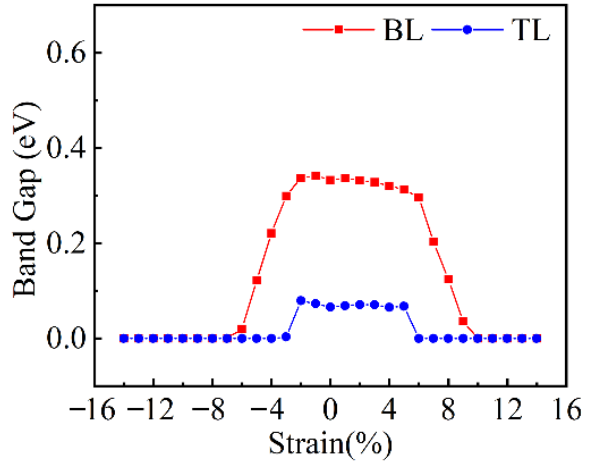

(b)

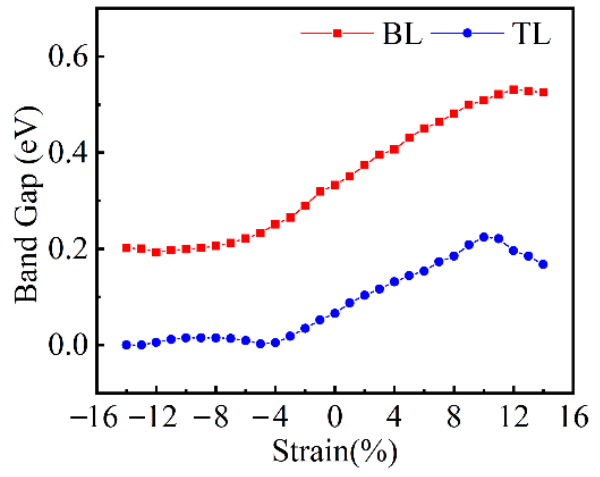

(c)

Figure 8. The variation in band gap of few-layer $\beta$-Te under uniaxial strain in different directions. (a) AC direction; (b) ZZ direction; (c) NM direction.

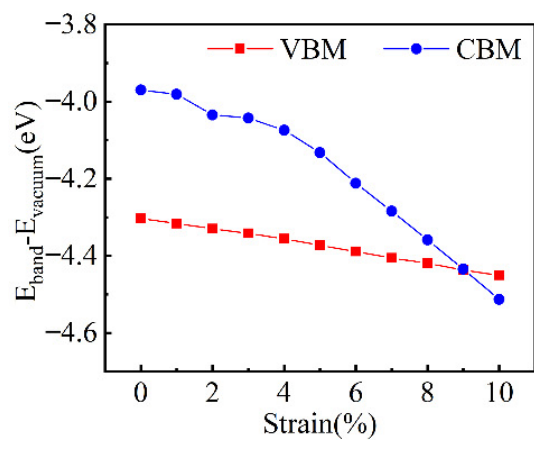

(a)

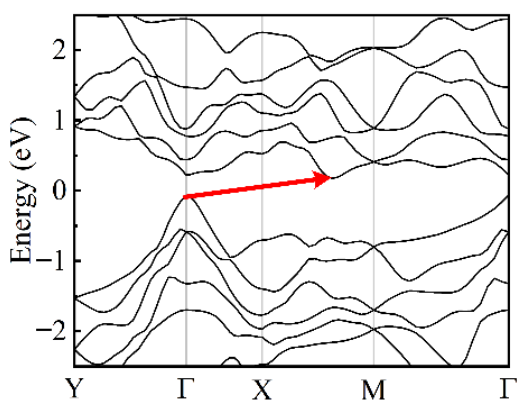

(b)

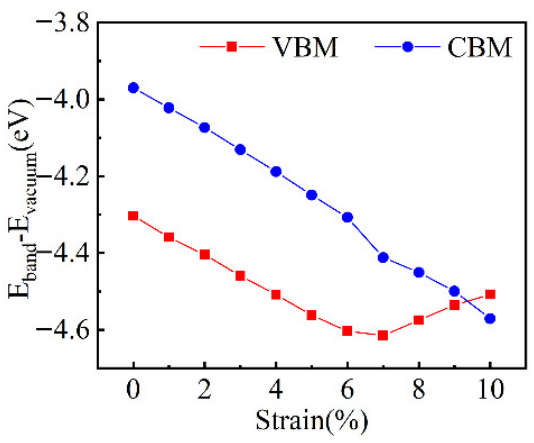

(c)

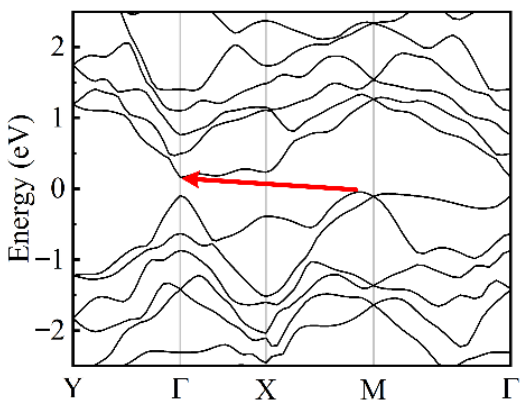

(d)



(e)



$(\mathbf{f})$

Figure 9. The variation in band structures of $B L \beta$-Te under uniaxial strain. The changes in the energy of VBM and CBM with tensile strains along the AC direction (a), and tensile (c) and compressive (e) strains along the ZZ direction. Band structures of BL $\beta$-Te under tensile strain of $5 \%$ (b) along the AC direction, and tensile strains of $7 \%(\mathbf{d})$ and compressive strain of $-3 \%$ (f) along the $\mathrm{ZZ}$ direction.

The summary of the effects of in-plane and out-of-plane uniaxial strain on the band gap of tellurene is shown in Table 2. Compared to the ML tellurene, the few-layer tellurene is more easily converted to metal. For both $\alpha$ and $\beta$ phases, the compressive deformation in $\mathrm{ZZ}$ is the most suitable to induce the SMT due to the lower critical strain. Moreover, it should be noted that the required compressive strain for the SMT in $\beta$-Te is smaller than $\alpha$-Te with the same layer, especially for thicker flakes. Hence, although the $\beta$-Te is hard to deform in the AC direction due to the higher YM, it becomes a metal more easily than the $\alpha$-Te. This indicates that a preprocess of converting $\alpha$ to $\beta$ tellurene by other methods, like charge doping, is also a promising way to prompt the SMT. The strain-induced SMT in fewlayer tellurene provides a fertile library for tuning the performance of materials, building 
metal-semiconductor junctions, and fabricating corresponding transistors. Besides the $\mathrm{SMT}$, the capacity of enlarging the band gap of few-layer tellurene showed by the strain in NM direction is also a promising way to modify the properties of nano-devices.

Table 2. Effects of in-plane and out-of-plane uniaxial strain on the band gap of tellurene.

\begin{tabular}{|c|c|c|c|c|c|c|c|}
\hline \multirow{2}{*}{ Phase } & \multirow{2}{*}{$\begin{array}{c}\text { Layer } \\
\text { Number }\end{array}$} & \multicolumn{2}{|c|}{ AC Direction } & \multicolumn{2}{|c|}{ ZZ Direction } & \multicolumn{2}{|c|}{ NM Direction } \\
\hline & & Tensile & Compressive & Tensile & Compressive & Tensile & Compressive \\
\hline \multirow{3}{*}{$\alpha-\mathrm{Te}$} & $\mathrm{BL}$ & SMT at $13 \%$ & decrease & slight & SMT at $-8 \%$ & decrease & \multirow{3}{*}{$\begin{array}{c}\text { decrease } \\
\text { increase then } \\
\text { decrease } \\
\text { increase then } \\
\text { decrease }\end{array}$} \\
\hline & $\mathrm{TL}$ & SMT at $11 \%$ & decrease & slight & SMT at $-7 \%$ & decrease & \\
\hline & FL & SMT at $10 \%$ & SMT at $-11 \%$ & slight & SMT at $-8 \%$ & decrease & \\
\hline \multirow{3}{*}{$\beta-\mathrm{Te}$} & ML & decrease & decrease & decrease & $\mathrm{SMT}$ at $-11 \%$ & decrease & \multirow{3}{*}{$\begin{array}{c}\text { / } \\
\text { increase then } \\
\text { decrease } \\
\text { increase then } \\
\text { decrease }\end{array}$} \\
\hline & $\mathrm{BL}$ & SMT at $10 \%$ & $\begin{array}{c}\text { increase then } \\
\text { decrease }\end{array}$ & SMT at $10 \%$ & SMT at $-7 \%$ & decrease & \\
\hline & TL & SMT at $4 \%$ & $\begin{array}{c}\text { increase then } \\
\text { decrease }\end{array}$ & SMT at $6 \%$ & SMT at $-4 \%$ & decrease & \\
\hline
\end{tabular}

\section{Conclusions}

In summary, the mechanical and electronic properties of $\alpha$ and $\beta$ tellurene under uniaxial strain were investigated by first-principles density functional theory calculations. The calculation results concerning the crystal structures reveal that the main difference between the $\alpha$-Te and $\beta$-Te is the location of the mid-Te atoms. The mid-Te atoms in $\beta$-Te locate at the symmetrical position and are bonded to the atoms in adjacent chains. Due to the bonds between the two Te atom chains, the $\beta$-Te exhibits dramatic higher YM and $\mathrm{PR}$ in the ZZ direction and similar performance in the AC direction, compared to the $\alpha$-Te. Hence, the $\alpha$-Te is more easily deformed by uniaxial strain in the ZZ direction. In addition, the variation in the atoms' configuration under uniaxial strain has showed that the in-plane compressive strain can induce the phase transition from $\alpha$ to $\beta$. However, no reverse transition is presented during in-plane and out-of-plane uniaxial deformation processes. For the instinct band structures, the few-layer $\alpha$-Te and $\beta$-Te have narrower band gaps as the layer increases, but the band gap of $\beta$-Te decays more dramatically than the $\alpha$-Te. Sequentially, the effects of uniaxial strain (in the range $-14 \% \leq \varepsilon \leq 14 \%$ ) on electronic properties were investigated. For the ML tellurene, the in-plane deformation induces the decrease in band gap. The layer compressive strain in the ZZ direction can induce the SMT. The strain-induced SMT was also observed in few-layer $\alpha$-Te and $\beta$-Te. Among different in-plane and out-of-plane deformations, compression in the ZZ direction is the most suitable approach to convert the tellurene to metal. The required transition strain in few-layer tellurene is lower than in ML and becomes lower as the thickness increases, especially for $\beta$-Te. The strain-induced SMT in tellurene provides a novel way to build metal-semiconductor junctions or even nano-devices in the same flakes without other metal electrode materials. In addition, although the normal strain is incapable of the SMT, the ability to enlarge the band gap greatly by tensile strain also has significant applications in controlling the performance of nano-devices. Therefore, strain-induced tuning of band structures in few-layer tellurene provides a foundation to design FETs, photodetectors, sensors, and memristors.

Supplementary Materials: The following are available online at https:/ /www.mdpi.com/article/10 $.3390 /$ nano12050875/s1, Table S1: Elastic stiffness constants, minimal and maximal YM and PR of $\alpha$-Te and $\beta$-Te, Figure S1: Mechanical properties of ML $\beta$ - Te, Figure S2: Mechanical properties of BL and FL tellurene, Figure S3: Band structures of ML $\beta-\mathrm{Te}$, Figure S4: Band structures of BL and FL tellurene, Figure S5: Band structures (left) and PDOSs (right) under the strains of $-11 \%$ along the ZZ direction, Figure S6: PDOS of Te atoms in ML $\beta$-Te, Figure S7: Band structures of ML $\beta$-Te under tensile strain along the AC direction, Figure S8: Band structures of FL $\beta$-Te under compressive 
strain of $-21 \%$ along the NM direction, Figure S9: Band structures of metallic TL $\alpha$-Te induced by uniaxial strains, Figure S10: PDOS of TL $\alpha$-Te under uniaxial strain, Figure S11: Band structures of metallic FL $\beta$-Te induced by uniaxial strain, Figure S12: Band structures of metallic BL $\beta$-Te induced by uniaxial strain, Figure S13: Band structure of BL $\beta$-Te under small uniaxial strain along the ZZ direction, Figure S14: Band structure of BL $\alpha$-Te under compressive strain of $-8 \%$ along the ZZ direction, Figure S15: PDOSs of BL $\beta$-Te under uniaxial strain, Figure S16: PDOS of Te atoms in BL $\beta$-Te under tensile strains of $7 \%$ along the $\mathrm{ZZ}$ direction.

Author Contributions: Conceptualization, G.W. and Y.D.; methodology, G.W.; software, G.W., Y.G. and L.Y.; validation, G.W., Y.D. and L.Y.; formal analysis, G.W.; investigation, G.W.; resources, G.W.; data curation, G.W.; writing-original draft preparation, G.W.; writing-review and editing, G.W. and Y.D.; visualization, G.W.; supervision, Y.W. and L.Y.; project administration, Y.D., Y.W. and L.Y.; funding acquisition, Y.D., Y.W. and L.Y. All authors have read and agreed to the published version of the manuscript.

Funding: National Natural Science Foundation of China, grant number 52105437; Heilongjiang Provincial Postdoctoral Science Foundation, grant number LBH-Z20054; and China Scholarship Council, grant number 202006120184.

Data Availability Statement: The data presented in this study are available on request from the corresponding author.

Acknowledgments: Genwang Wang acknowledges the support given by Kah-Wee Ang at the National University of Singapore.

Conflicts of Interest: The authors declare no conflict of interest.

\section{References}

1. Mannix, A.J.; Kiraly, B.; Hersam, M.C.; Guisinger, N.P. Synthesis and chemistry of elemental 2D materials. Nat. Rev. Chem. 2017, 1, 14. [CrossRef]

2. Yang, Z.; Hao, J. Progress in pulsed laser deposited two-dimensional layered materials for device applications. J. Mater. Chem. C 2016, 4, 8859-8878. [CrossRef]

3. Wang, G.; Hou, C.; Long, H.; Yang, L.; Wang, Y. Electronic and optoelectronic nanodevices based on two-dimensional semiconductor materials. Acta Phys.-Chim. Sin. 2019, 35, 1319-1340. [CrossRef]

4. Long, M.; Wang, P.; Fang, H.; Hu, W. Progress, challenges, and opportunities for 2D material based photodetectors. Adv. Funct. Mater. 2019, 29, 1803807. [CrossRef]

5. Yang, D.; Yang, R.; Priya, S.; Liu, S. Recent advances in flexible perovskite solar cells: Fabrication and applications. Angew. Chem. Int. Ed. 2019, 58, 4466-4483. [CrossRef]

6. Li, Y.; Ang, K.W. Hardware Implementation of Neuromorphic Computing Using Large-Scale Memristor Crossbar Arrays. Adv. Intell. Syst. 2021, 3, 2000137. [CrossRef]

7. Liu, X.; Ren, J.C.; Shen, T.; Li, S.; Liu, W. Lateral InSe p-n Junction Formed by Partial Doping for Use in Ultrathin Flexible Solar Cells. J. Phys. Chem. Lett. 2019, 10, 7712-7718. [CrossRef]

8. Prakash, A.; Cai, Y.; Zhang, G.; Zhang, Y.W.; Ang, K.W. Black Phosphorus N-Type Field-Effect Transistor with Ultrahigh Electron Mobility via Aluminum Adatoms Doping. Small 2017, 13, 1602909. [CrossRef]

9. Qi, J.; Li, X.; Qian, X.; Feng, J. Bandgap engineering of rippled $\mathrm{MoS}_{2}$ monolayer under external electric field. Appl. Phys. Lett. 2013, 102, 173112. [CrossRef]

10. Nguyen, C.V.; Hieu, N.N.; Ilyasov, V.V. Band gap modulation of bilayer $\mathrm{MoS}_{2}$ under strain engineering and electric field: A density functional theory. J. Electron. Mater. 2016, 45, 4038-4043. [CrossRef]

11. Li, Z.; Chen, F. Ion beam modification of two-dimensional materials: Characterization, properties, and applications. Appl. Phys. Rev. 2017, 4, 011103. [CrossRef]

12. Novoselov, K.S.; Mishchenko, O.A.; Carvalho, O.A.; Neto, A.C. 2D materials and van der Waals heterostructures. Science 2016, 353, acc9439. [CrossRef] [PubMed]

13. Liu, Y.; Weiss, N.O.; Duan, X.; Cheng, H.C.; Huang, Y.; Duan, X. Van der Waals heterostructures and devices. Nat. Rev. Mater. 2016, 1, 16042. [CrossRef]

14. Cao, K.; Feng, S.; Han, Y.; Gao, L.; Ly, T.H.; Xu, Z.; Lu, Y. Elastic straining of free-standing monolayer graphene. Nat. Commun. 2020, 11, 284. [CrossRef]

15. Akinwande, D.; Brennan, C.J.; Bunch, J.S.; Egberts, P.; Felts, J.R.; Gao, H.; Zhu, Y. A review on mechanics and mechanical properties of 2D materialls-Graphene and beyond. Extrem. Mech. Lett. 2017, 13, 42-77. [CrossRef]

16. Bao, H.; Huang, Y.; Yang, Z.; Miao, Y.; Chu, P.K.; Xu, K.; Ma, F. Tensile loading induced phase transition and rippling in single-layer $\mathrm{MoS}_{2}$. Appl. Surf. Sci. 2017, 404, 180-187. [CrossRef] 
17. Manzeli, S.; Allain, A.; Ghadimi, A.; Kis, A. Piezoresistivity and strain-induced band gap tuning in atomically thin $\mathrm{MoS}_{2}$. Nano Lett. 2015, 15, 5330-5335. [CrossRef]

18. Chen, Y.; Deng, W.; Chen, X.; Wu, Y.; Shi, J.; Zheng, J.; Zhang, Y. Carrier mobility tuning of MoS 2 by strain engineering in CVD growth process. Nano Res. 2021, 14, 2314-2320. [CrossRef]

19. Yu, L.; Ruzsinszky, A.; Perdew, J.P. Bending two-dimensional materials to control charge localization and Fermi-level shift. Nano Lett. 2016, 16, 2444-2449. [CrossRef]

20. Chen, S.; Sood, A.; Pop, E.; Goodson, K.E.; Donadio, D. Strongly tunable anisotropic thermal transport in $\mathrm{MoS}_{2}$ by strain and lithium intercalation: First-principles calculations. 2D Mater. 2019, 6, 025033. [CrossRef]

21. Liu, T.; Liu, S.; Tu, K.H.; Schmidt, H.; Chu, L.; Xiang, D.; Martin, J.; Eda, G.; Ross, C.A.; Garaj, S. Crested two-dimensional transistors. Nat. Nanotechnol. 2019, 14, 223-226. [CrossRef] [PubMed]

22. Guo, J.; Wen, R.; Zhai, J.; Wang, Z.L. Enhanced $\mathrm{NO}_{2}$ gas sensing of a single-layer $\mathrm{MoS}_{2}$ by photogating and piezo-phototronic effects. Sci. Bull. 2019, 64, 128-135. [CrossRef]

23. Lin, P.; Pan, C.; Wang, Z.L. Two-dimensional nanomaterials for novel piezotronics and piezophototronics. Mater. Today Nano 2018, 4, 17-31. [CrossRef]

24. Zhu, Z.; Cai, X.; Yi, S.; Chen, J.; Dai, Y.; Niu, C.; Guo, Z.; Xie, M.; Liu, F.; Cho, J.H.; et al. Multivalency-driven formation of Te-based monolayer materials: A combined first-principles and experimental study. Phys. Rev. Lett. 2017, 119, 106101. [CrossRef] [PubMed]

25. Wang, Y.; Xiao, C.; Chen, M.; Hua, C.; Zou, J.; Wu, C.; Jiang, J.; Yang, S.A.; Ji, W. Two-dimensional ferroelectricity and switchable spin-textures in ultra-thin elemental Te multilayers. Mater. Horiz. 2018, 5, 521-528. [CrossRef]

26. Xiang, Y.; Gao, S.; Xu, R.G.; Wu, W.; Leng, Y. Phase transition in two-dimensional tellurene under mechanical strain modulation Nano Energy 2019, 58, 202-210. [CrossRef]

27. Wang, Y.; Qiu, G.; Wang, R.; Huang, S.; Wang, Q.; Liu, Y.; Du, Y.; Goddard, W.A.; Kim, M.J.; Xu, X.; et al. Field-effect transistors made from solution-grown two-dimensional tellurene. Nat. Electron. 2018, 1, 228-236. [CrossRef]

28. Xie, Z.; Xing, C.; Huang, W.; Fan, T.; Li, Z.; Zhao, J.; Xiang, Y.; Guo, Z.; Li, J.; Yang, Z.; et al. Ultrathin 2D nonlayered tellurium nanosheets: Facile liquid-phase exfoliation, characterization, and photoresponse with high performance and enhanced stability. Adv. Funct. Mater. 2018, 28, 1705833. [CrossRef]

29. Chen, J.; Dai, Y.; Ma, Y.; Dai, X.; Ho, W.; Xie, M. Ultrathin $\beta$-tellurium layers grown on highly oriented pyrolytic graphite by molecular-beam epitaxy. Nanoscale 2017, 9, 15945-15948. [CrossRef]

30. Apte, A.; Bianco, E.; Krishnamoorthy, A.; Yazdi, S.; Rao, R.; Glavin, N.; Kumazoe, H.; Varshney, V.; Roy, A.; Shimojo, F.; et al. Polytypism in ultrathin tellurium. 2D Mater. 2018, 6, 015013. [CrossRef]

31. Yang, S.; Chen, B.; Qin, Y.; Zhou, Y.; Liu, L.; Durso, M.; Zhuang, H.; Shen, Y.; Tongay, S. Highly crystalline synthesis of tellurene sheets on two-dimensional surfaces: Control over helical chain direction of tellurene. Phys. Rev. Mater. 2018, 2, 104002. [CrossRef]

32. Amani, M.; Tan, C.; Zhang, G.; Zhao, C.; Bullock, J.; Song, X.; Kim, H.; Shrestha, V.R.; Gao, Y.; Crozier, K.B.; et al. Solutionsynthesized high-mobility tellurium nanoflakes for short-wave infrared photodetectors. ACS Nano 2018, 12, 7253-7263. [CrossRef] [PubMed]

33. Wang, D.; Yang, A.; Lan, T.; Fan, C.; Pan, J.; Liu, Z.; Chu, J.; Yuan, H.; Wang, X.; Rong, M.; et al. Tellurene based chemical sensor. J. Mater. Chem. A 2019, 7, 26326-26333. [CrossRef]

34. Dong, Y.; Zeng, B.; Zhang, X.; Li, D.; He, J.; Long, M. Study on the strain-induced mechanical property modulations in monolayer Tellurene. J. Appl. Phys. 2019, 125, 064304. [CrossRef]

35. Ma, H.; Hu, W.; Yang, J. Control of highly anisotropic electrical conductance of tellurene by strain-engineering. Nanoscale 2019, 11, 21775-21781. [CrossRef]

36. Sopiha, K.V.; Malyi, O.I.; Persson, C. First-principles mapping of the electronic properties of two-dimensional materials for strain-tunable nanoelectronics. ACS Appl. Nano Mater. 2019, 2, 5614-5624. [CrossRef]

37. Wang, Y.; Yao, S.; Liao, P.; Jin, S.; Wang, Q.; Kim, M.J.; Cheng, G.J.; Wu, W. Strain-Engineered Anisotropic Optical and Electrical Properties in 2D Chiral-Chain Tellurium. Adv. Mater. 2020, 32, 2002342. [CrossRef]

38. Gao, S.; Wang, Y.; Wang, R.; Wu, W. Piezotronic effect in 1D van der Waals solid of elemental tellurium nanobelt for smart adaptive electronics. Semicond. Sci. Technol. 2017, 32, 104004. [CrossRef]

39. Du, Y.; Qiu, G.; Wang, Y.; Si, M.; Xu, X.; Wu, W.; Ye, P.D. One-dimensional van der Waals material tellurium: Raman spectroscopy under strain and magneto-transport. Nano Lett. 2017, 17, 3965-3973. [CrossRef]

40. Ju, W.; Li, T.; Wang, H.; Yong, Y.; Li, X. Hybrid functional studies on the electronic properties of ultrathin black phosphorus under normal strain. Comput. Mater. Sci. 2015, 109, 20-24. [CrossRef]

41. Castellanos-Gomez, A.; Roldán, R.; Cappelluti, E.; Buscema, M.; Guinea, F.; van der Zant, H.S.; Steele, G.A. Local strain engineering in atomically thin $\mathrm{MoS}_{2}$. Nano Lett. 2013, 13, 5361-5366. [CrossRef] [PubMed]

42. Kohn, W.; Sham, L.J. Self-consistent equations including exchange and correlation effects. Phys. Rev. 1965, 140, A1133. [CrossRef]

43. Giannozzi, P.; Baroni, S.; Bonini, N.; Calandra, M.; Car, R.; Cavazzoni, C.; Ceresoli, D.; Chiarotti, G.L.; Cococcioni, M.; Dabo, I.; et al. QUANTUM ESPRESSO: A modular and open-source software project for quantum simulations of materials. J. Phys. Condens. Matter 2009, 21, 395502. [CrossRef] [PubMed]

44. Burke, K.; Perdew, J.P.; Wang, Y. Derivation of a generalized gradient approximation: The PW91 density functional. In Electronic Density Functional Theory; Springer: Boston, MA, USA, 1998; pp. 81-111. 
45. Grimme, S. Semiempirical GGA-type density functional constructed with a long-range dispersion correction. J. Comput. Chem. 2006, 27, 1787-1799. [CrossRef]

46. Wang, L.; Kutana, A.; Zou, X.; Yakobson, B.I. Electro-mechanical anisotropy of phosphorene. Nanoscale 2015, 7, 9746-9751. [CrossRef]

47. Zhou, J.; Huang, R. Internal lattice relaxation of single-layer graphene under in-plane deformation. J. Mech. Phys. Solids 2008, 56, 1609-1623. [CrossRef]

48. Zhuang, H.L.; Hennig, R.G. Computational search for single-layer transition-metal dichalcogenide photocatalysts. J. Phys. Chem. C 2013, 117, 20440-20445. [CrossRef]

49. Cahangirov, S.; Topsakal, M.; Aktürk, E.; Şahin, H.; Ciraci, S. Two-and one-dimensional honeycomb structures of silicon and germanium. Phys. Rev. Lett. 2009, 102, 236804. [CrossRef]

50. Cai, X.; Ren, Y.; Wu, M.; Xu, D.; Luo, X. Strain-induced phase transition and giant piezoelectricity in monolayer tellurene. Nanoscale 2020, 12, 167-172. [CrossRef] [PubMed]

51. Wang, C.; Zhou, X.; Qiao, J.; Zhou, L.; Kong, X.; Pan, Y.; Cheng, Z.; Chai, Y.; Ji, W. Charge-governed phase manipulation of few-layer tellurium. Nanoscale 2018, 10, 22263-22269. [CrossRef] 